\title{
The association between the consumption of eggs and psychological distress in Japanese adults
}

\author{
A. Nishide ${ }^{1}$, Y. Sato ${ }^{2}$ and T. Goto ${ }^{3}$ \\ ${ }^{1}$ User Life Science Co. Ltd. Japan, \\ ${ }^{2}$ Epidemiology Research Group, National Institute of Occupational Safety and Health, Japan and \\ ${ }^{3}$ Faculty of Human Life Science, Miyagi Gakuin Women's University, Japan
}

A previous study reported that after the Great East Japan Earthquake of 2011, the prevalence of adults presenting with psychological distress was nearly $50 \%{ }^{(1)}$ in the city in north Japan. A diet, rich in fish $^{(2)}$ and plant proteins, including soy ${ }^{(3)}$, is reported to have a beneficial effect on mental health conditions. However, little research has been undertaken on the consumption of egg, despite this food being one of the most important sources of protein, all essential amino acids, including tryptophan; a precursor for serotonin, as well as B Vitamins and n-3 fatty acids. A previous study, focusing on daily egg consumption in Iran, failed to detect the effect of egg intake on mental health conditions and concluded that this was due to differences in the energy levels of individuals. ${ }^{(4)}$ Therefore, we observed the association between egg intake, calculating the amount per 1,000 kcal, and mental health. Thus, the aim of this study is to assess the relationship between the consumption of eggs, calculated in grams per 1,000 kcal, and the prevalence of psychological distress in Japanese adults living in an area affected by the natural disaster of 2011.

In March 2017, a self-administered questionnaire survey, including a Kessler Psychological Distress Scale (K6) to evaluate the level of psychological distress and a Food Frequency Questionnaire consisting of 185 different items were distributed to the 60 adult volunteers. The response data of 58 respondents was used in the analysis. A cut off score of five was applied to identify cases of psychological distress. Furthermore, binary logistic regression was employed to estimate the odds ratios (ORs) and $95 \%$ confidence intervals for psychological distress across the tertile of egg consumption, calculated in grams per $1000 \mathrm{kcal}$, in crude and multivariable-adjusted models when $\mathrm{p}<0.05$ was considered statistically significant.

A lower OR of psychological distress (k6> =5) was found in the bottom tertile of the egg consumption group (14.3 g/1000 kcal-16.7 $\mathrm{g} / 1000 \mathrm{kcal}$. This remained unchanged after adjustments were made for gender, age, and the number of years spent in education. Significant differences of some nutrient intakes among the egg consumption groups were observed.

Our findings were similar to those of the previous study which reported it was challenging to determine the effect of egg consumption on psychological conditions due to the interaction of other nutrient factors.

1. Niitsu T, Takaoka K, Uemura S et al. (2014) BMC, 307.

2. Timonen M, Horrobin D, Jokelainen J et al. (2004) J Affect Disord 82, 447-452.

3. Yu S, Guo X, Yang H et al. (2015) J Nutr Health Aging 19,884-893.

4. Shafiel F, Keshteli AH et al. (2018) Eur J Nutr 58, 1923-1932. 